\title{
Propellant Mass Fraction Calculation Methodology for Launch Vehicles and Application to Ares Vehicles
}

\author{
James B. Holt ${ }^{1}$ and Timothy S. Monk ${ }^{2}$ \\ NASA Marshall Space Flight Center, Huntsville, AL 35812, USA
}

\begin{abstract}
Propellant Mass Fraction (pmf) calculation methods vary throughout the aerospace industry. While typically used as a means of comparison between candidate launch vehicle designs, the actual pmf calculation method varies slightly from one entity to another. It is the purpose of this paper to present various methods used to calculate the pmf of launch vehicles. This includes fundamental methods of pmf calculation that consider only the total propellant mass and the dry mass of the vehicle; more involved methods that consider the residuals, reserves and any other unusable propellant remaining in the vehicle; and calculations excluding large mass quantities such as the installed engine mass. Finally, a historical comparison is made between launch vehicles on the basis of the differing calculation methodologies, while the unique mission and design requirements of the Ares $\mathbf{V}$ Earth Departure Stage (EDS) are examined in terms of impact to pmf.
\end{abstract}

Nomenclature

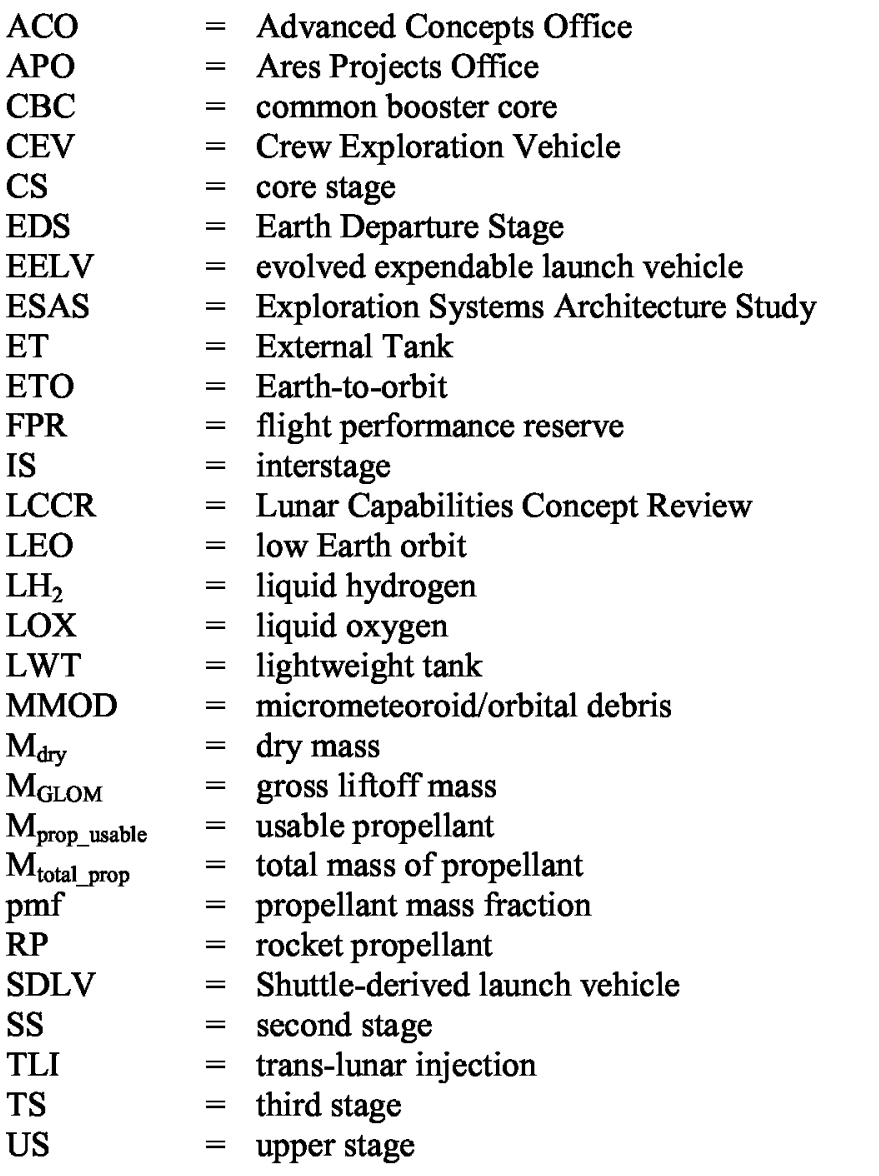

\footnotetext{
${ }_{1}^{1}$ Aerospace Engineer, Advanced Concepts Office, ED04 NASA MSFC, Huntsville, AL 35812.

${ }^{2}$ Systems Integration Engineer, Zero Point Frontiers Corp., 2105 Shades Crest Rd. SE, Huntsville, AL 35801.
} 


\section{Introduction}

There are several mass fractions that can be calculated for launch vehicle designs. These include the structural mass fraction, mass ratios at a system or subsystem level, and the propellant mass fraction (pmf). These mass fractions or mass ratios are utilized in different manners by the various engineering disciplines. A very simple calculation to assess the structural efficiency of a launch vehicle design, gauge conservatism in a design, or to compare candidate designs is the pmf. As applied to launch vehicle stages, the pmf describes the ratio of propellant in a given stage to the total stage mass. When a consistent methodology for this calculation is employed, it can be very useful to designers of launch vehicles, launch vehicle stages, or even at the subsystem level. However, there are various methods for performing this calculation, and its meaning varies across the industry. Presented are methods for calculating pmf (as applied to launch vehicle stages in this case), a comparison of selected launch vehicle stages, and the impact on the pmf of mission requirements currently facing the Ares V Earth Departure Stage (EDS).

\section{Fundamental pmf Calculation}

A basic method for calculating pmf that is widely used in undergraduate textbooks or when limited information is available involves only two known variables - total propellant mass of a given stage $\left(\mathrm{M}_{\text {total prop }}\right)$ and the total dry mass of the stage $\left(\mathrm{M}_{\mathrm{dry}}\right)$. Using these two variables, the pmf is calculated as:

$$
p m f=\frac{M_{\text {total_prop }}}{M_{\text {total_prop }}+M_{d r y}}
$$

Stated otherwise, the denominator in this equation is the total mass of the stage. Therefore, the equation is understood to be the total propellant mass divided by the gross liftoff mass $\left(\mathrm{M}_{\mathrm{GLOM}}\right)$ of the stage. Although used widely, this method fails to account for several realistic assumptions, including liquid propellant residuals, reserves, and others, which are discussed later.

As an example, the Lunar Capabilities Concept Review (LCCR) Ares V vehicle (also known as the 51.00 .48 configuration) is comprised of two liquid propellant stages and two solid rocket boosters. The liquid propellant stages are known as the Core Stage (CS) and the EDS. The Ares V vehicle is shown in Fig. 1.

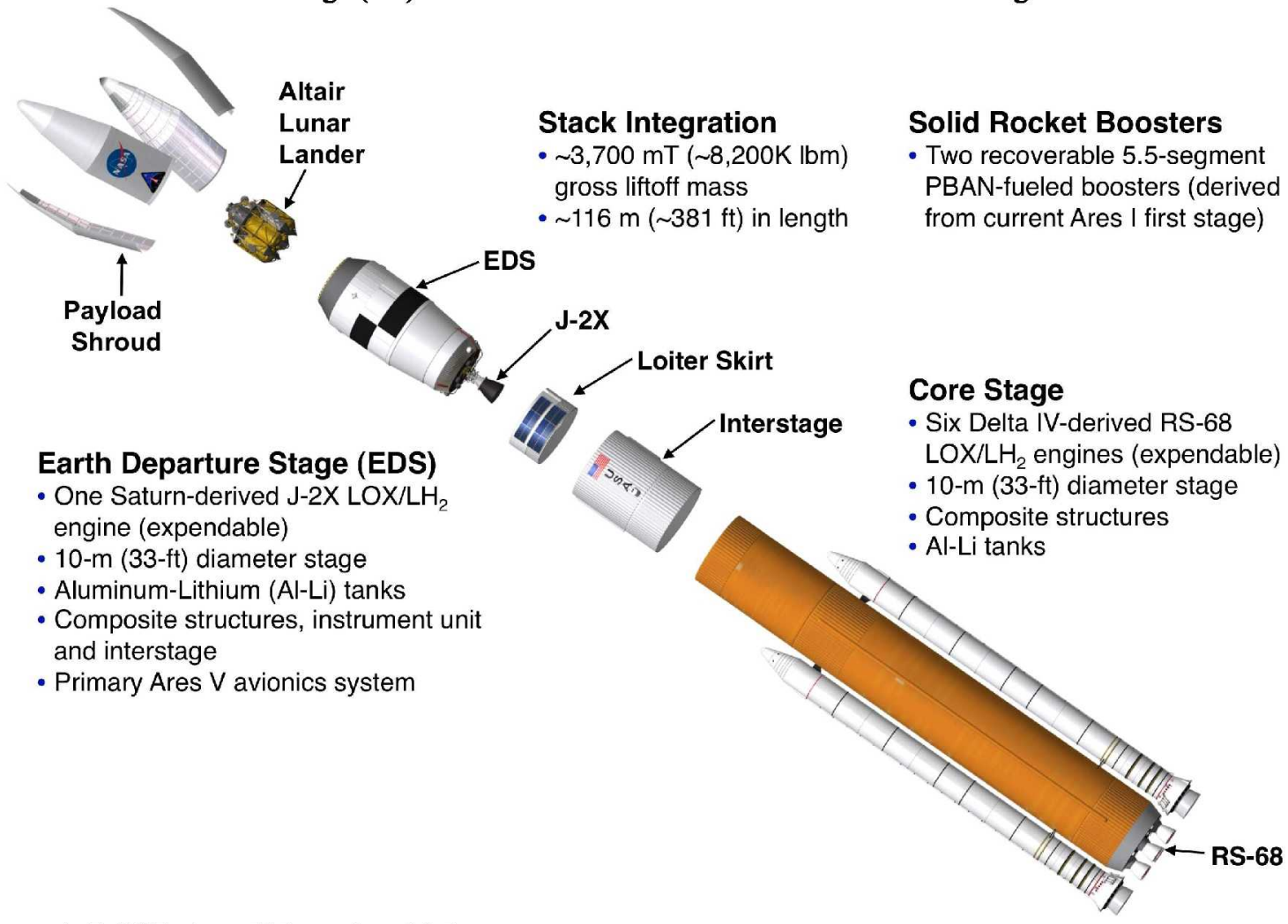

Figure 1. LCCR Ares V launch vehicle. 
The EDS has a total propellant capacity of approximately $579,000 \mathrm{lb}_{\mathrm{m}}$ and a dry mass of approximately 53,000 $\mathrm{lb}_{\mathrm{m}}$. Using Eq. (1), the pmf of the Ares V EDS would be calculated as follows:

$$
p m f=\frac{579,000}{579,000+53,000}=0.916
$$

\section{Current Methodology of the MSFC Advanced Concepts Office}

The Advanced Concepts Office (ACO) at NASA Marshall Space Flight Center (MSFC) has performed thousands of vehicle trades from the time period leading up to the Exploration Systems Architecture Study (ESAS) until present. These vehicle trades include options ranging from clean sheet designs to the Evolved Expendable Launch Vehicles (EELVs) and Shuttle Derived Launch Vehicles (SDLVs) - which includes both sidemount and inline vehicle options. A primary means of comparison among these designs is the structural efficiency of the vehicle stages captured by the pmf calculation. The method employed for this calculation is very simple and is used in several respected publications. ${ }^{1-3^{*}}$ It includes only two terms - the total usable propellant mass of the stage ( $\left.\mathrm{M}_{\text {propUsable }}\right)$ and the gross liftoff mass $\left(\mathrm{M}_{\mathrm{GLOM}}\right)$ of the stage. The pmf is then found as follows:

$$
p m f=\frac{M_{\text {prop Usable }}}{M_{\text {GLOM }}}
$$

The usable propellant mass differs from the total propellant capacity in that it accounts for the propellant mass allocated for several realistic conditions. Among these is the propellant allocated for the flight performance reserve (FPR), fuel bias, liquid residuals, engine restart, purges/bleeds, boiloff of cryogenic propellants, and other losses. The FPR is the propellant allocated in the final stage of the vehicle to account for 3-sigma ascent performance dispersions, day-of-flight off-nominal conditions, etc. The fuel bias is a specified amount of fuel dedicated to making sure that the maximum amount of oxidizer is used for propulsive maneuvers. This protects against uncertainties associated with cryogenic propellant loading, off-nominal mixture ratio dispersions, and avoids an undesirable LOX-rich engine shutdown condition. Nevertheless, a finite amount of propellant is left in the oxidizer and fuel feedlines, turbopumps, and other propellant feed system components at engine cutoff. The allocation of propellant for this is termed liquid residuals. Furthermore, propellant is needed for engine restarts, purges/bleeds if necessary, boiloff of the cryogenic propellants if any extended time on-orbit is required, or other in-flight fluid losses.

Using the LCCR Ares V vehicle again as an example, it is calculated that the total usable propellant in the EDS is $\sim 558,000 \mathrm{lb}_{\mathrm{m}}$, while the GLOM of the stage is the same $632,000 \mathrm{lb}_{\mathrm{m}}$ as previously calculated. Therefore, a more realistic calculation of pmf is as follows:

$$
p m f=\frac{558,000}{632,000}=0.883
$$

\section{Large Mass Exclusion}

Another common practice for calculating pmf involves excluding large quantities of mass from $\mathrm{M}_{\mathrm{GLOM}}$. These large mass exclusions typically include either the mass of an interstage, if applicable, or the installed engine mass. For instance, during the Saturn V program, the mass of the interstage connecting the S-IC stage to the S-II stage was tracked along with the S-II stage. Likewise, the interstage connecting the S-II stage to the S-IVB stage was tracked with the S-IVB stage. While there will be an obvious negative impact to the pmf calculation of these stages if the interstage is included, it is a more accurate depiction of the stage pmf to include these quantities. Nevertheless, it is useful at times to compare stages of differing designs, engines, etc. without these large quantities.

Table 1. Ares $V$ core stage pmf comparison.

\begin{tabular}{|l|c|c|c|}
\hline & Usable Prop Mass $\left(\mathrm{lb}_{\mathrm{m}}\right)$ & GLOM $\left(\mathrm{lb}_{\mathrm{m}}\right)$ & pmf \\
\hline Core Stage w/ Interstage & $\sim 3,500,000$ & $\sim 3,902,000$ & 0.897 \\
\hline Core Stage w/o Interstage & $\sim 3,500,000$ & $\sim 3,882,000$ & 0.902 \\
\hline
\end{tabular}

\footnotetext{
* Reference 1, while not using the same equation as (2), references the fact that an assumption of complete propellant depletion will never be satisfied exactly. That is, there will be some liquid residuals or intentional propellant allocations that should be accounted for.
} 
For example, the LCCR Ares V launch vehicle has an interstage of approximately $20,000 \mathrm{lb}_{\mathrm{m}}$. Through LCCR the interstage mass was tracked with the CS of the Ares V. The total GLOM of the CS excluding the interstage is approximately $3,882,000 \mathrm{lb}_{\mathrm{m}}$, while the propellant mass of the CS is approximately $3,500,000 \mathrm{lb}_{\mathrm{m}}$. Table 1 shows the pmf of the CS both with and without the interstage included in the calculation.

The engine mass is sometimes excluded from the total mass of the stage when comparing designs due to the wide range of mass associated with liquid rocket engines. An example of this can be found in a comparison of the Saturn V S-IVB stage to the Upper Stage (US) of the Ares I launch vehicle which is compared in Fig. 2. These two stages share a common bulkhead tank design, utilize a single J-2 class engine, and have reasonably similar diameters \& propellant masses. However, the difference in mass from the J-2 utilized on the S-IVB \& the J-2X currently being developed for the Ares I US is on the order of $2,000 \mathrm{lb}_{\mathrm{m}}$ due to the increased expansion ratio of the current $\mathrm{J}-2 \mathrm{X}$ design.

In the case of these similar stages, a comparison is helpful for both the calculation of pmf with total GLOM $\left(\mathrm{pmf}_{1}\right)$ and without the installed engine mass $\left(\mathrm{pmf}_{2}\right)$. All of the calculations also exclude the interstage mass. The pmf difference between the S-IVB and Ares I US is approximately 0.006 when including the engine mass $\left(\mathrm{pmf}_{1}\right)$, but the difference in pmf is reduced to $\sim 0.003$ when excluding the engine mass $\left(\mathrm{pmf}_{2}\right)$ as can be seen in Table 2. Overall, this comparison confirms that the Ares I US has a historical precedent for the calculated pmf value.

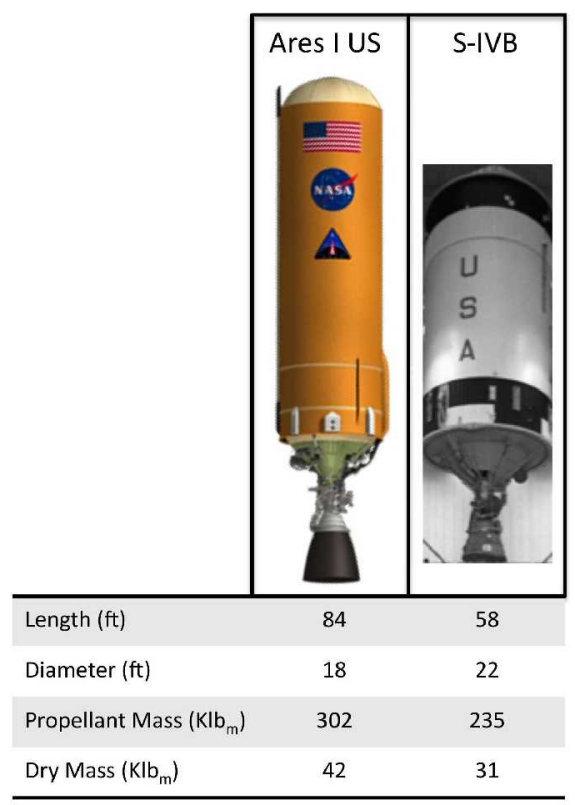

Figure 2. Similar stage comparison.

Table 2. Saturn S-IVB and Ares I US pmf comparison.

\begin{tabular}{|l|c|c|c|c|c|}
\hline & Usable Prop Mass $\left(\mathrm{lb}_{\mathrm{m}}\right)$ & GLOM $\left(\mathrm{lb}_{\mathrm{m}}\right)$ & GLOM w/o Engine $\left(\mathrm{lb}_{\mathrm{m}}\right)$ & pmf $_{1}$ & pmf $_{2}$ \\
\hline Saturn S-IVB & $\sim 235,000$ & $\sim 266,000$ & $\sim 262,500$ & 0.884 & 0.895 \\
\hline Ares I US & $\sim 302,000$ & $\sim 344,000$ & $\sim 338,600$ & 0.878 & 0.892 \\
\hline
\end{tabular}

\section{Historical pmf Comparison}

To place the Ares I and Ares V launch vehicles into a historical context can be useful to gauge the efficiency of their designs and/or identify key drivers of their design and its impact on overall performance. The pmf of selected launch vehicle upper stages is shown in Fig. 3 as a function of usable propellant mass. The Ares I US and Saturn SIVB and S-II stages are shown both with and without their respective interstage. In addition, the Ares V EDS is depicted in the baseline lunar sortie configuration (labeled 51.00.48 EDS) which requires a 4 day loiter in Low Earth Orbit (LEO), a J-2X engine restart, etc. The "51.01.48 EDS - Lunar Cargo" case does not require a loiter period however, since it targets the Moon directly with no intermediate parking orbit in LEO. This is a very large performance driver that has a great impact on pmf, as shown. Furthermore, among NASA designed stages the expected trend is very noticeable - there is a gradual increase in pmf as propellant load increases.

Figure 4 depicts the pmf of first stages, booster stages, and core stages. The most common propellant for these stages is LOX/Rocket Propellant (RP) due to the higher density propellants and more thrust available from these liquid rocket engines. The only $\mathrm{LOX} / \mathrm{LH}_{2}$ stages depicted in Figure 4 are the Delta IV Common Booster Core (CBC), the Ares V CS, the Ariane 5 CS, and the Space Shuttle External Tank (ET). The Space Shuttle ET has three data points: the original ET, the light-weight tank (LWT), and the super light-weight tank (SLWT). As expected, the pmf calculations of these external tank data points are greater than any other data point due to these tanks not having engines, thrust structures, integrated thrust loads from the engines, and other subsystems found on the other stages depicted.

It can be seen that the higher density of the LOX/RP propellants allow for a very efficient structural design. As can be expected, the higher propellant load of the Ares V CS allows for a higher pmf than the lower propellant mass Delta IV CBC. However, the H158 Ariane 5G CS has a very high pmf for a LOX/LH 2 rocket stage. This is due to its common bulkhead design and very low thrust load for a core stage (approximately $256,000 \mathrm{lb}_{\mathrm{f}}$ from a single Vulcain engine). 


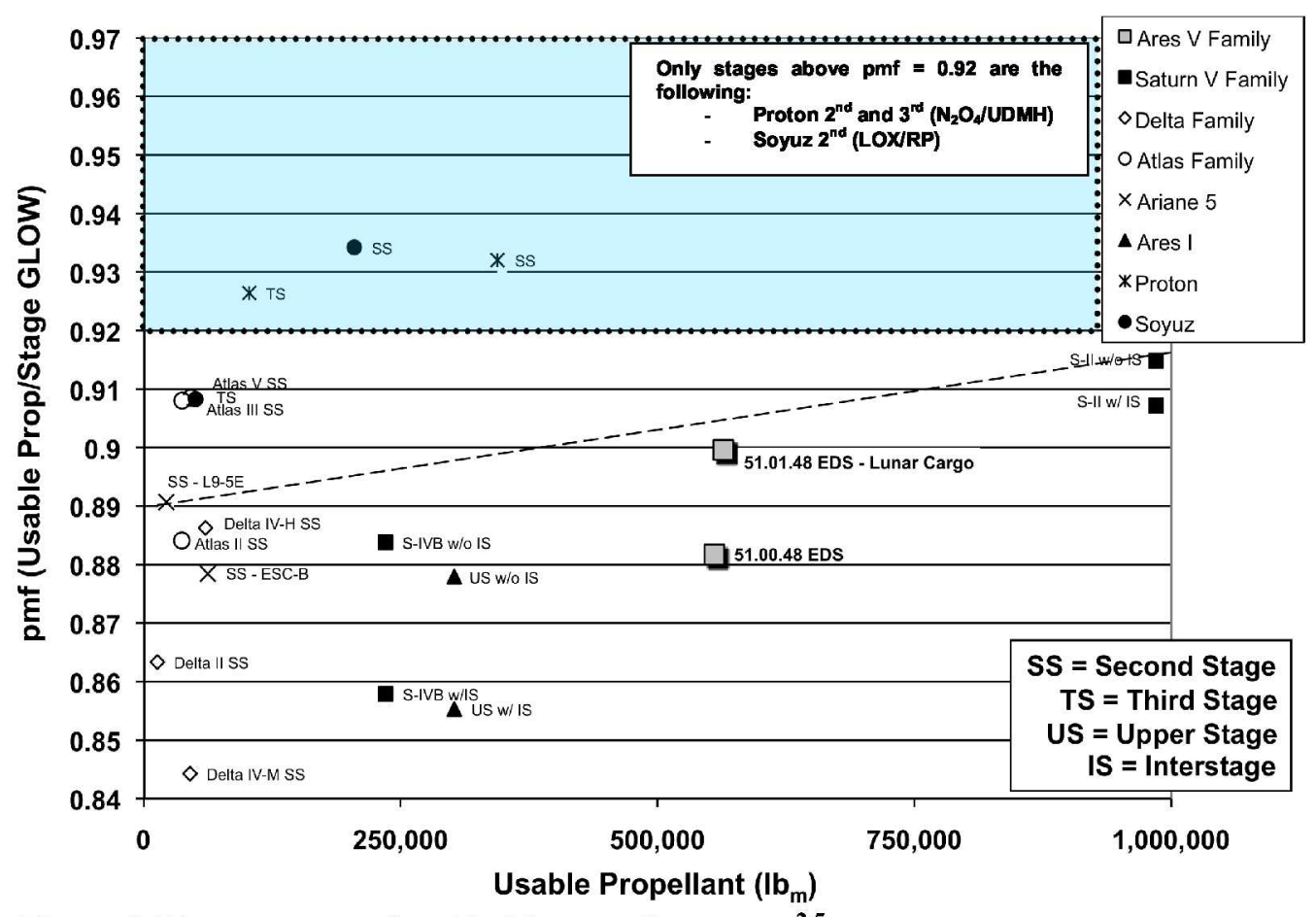

Figure 3. Upper stage pmf vs. Usable propellant mass. ${ }^{3-5}$

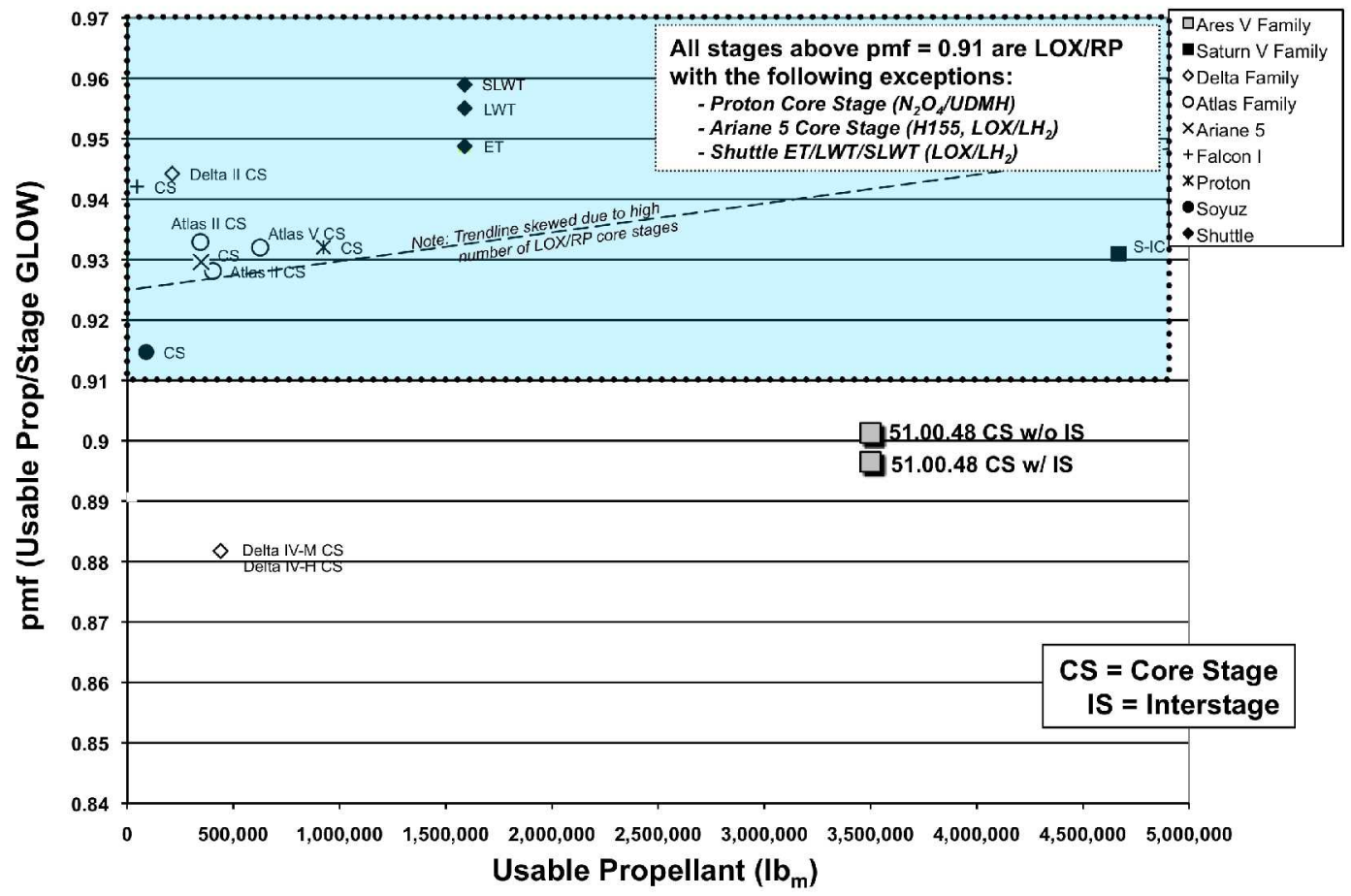

Figure 4. Core stage pmf vs. Usable propellant mass. ${ }^{3-5}$

Most of the data shown in Figures 3-5 can be further examined in International Reference Guide to Space Launch Systems [3], while the Saturn V data points are derived from the Apollo 15 Flight Evaluation Report [4]. The Ares I \& V data points are provided by the MSFC Ares Projects Office [5]. 
Figure 5 depicts flown $\mathrm{LOX} / \mathrm{LH}_{2}$ Centaur stages, Saturn V launch vehicle stages, and the Ares $\mathrm{V}$ stages as a function of calendar year. It can be clearly seen that there has been a positive trend in pmf (and thus structural

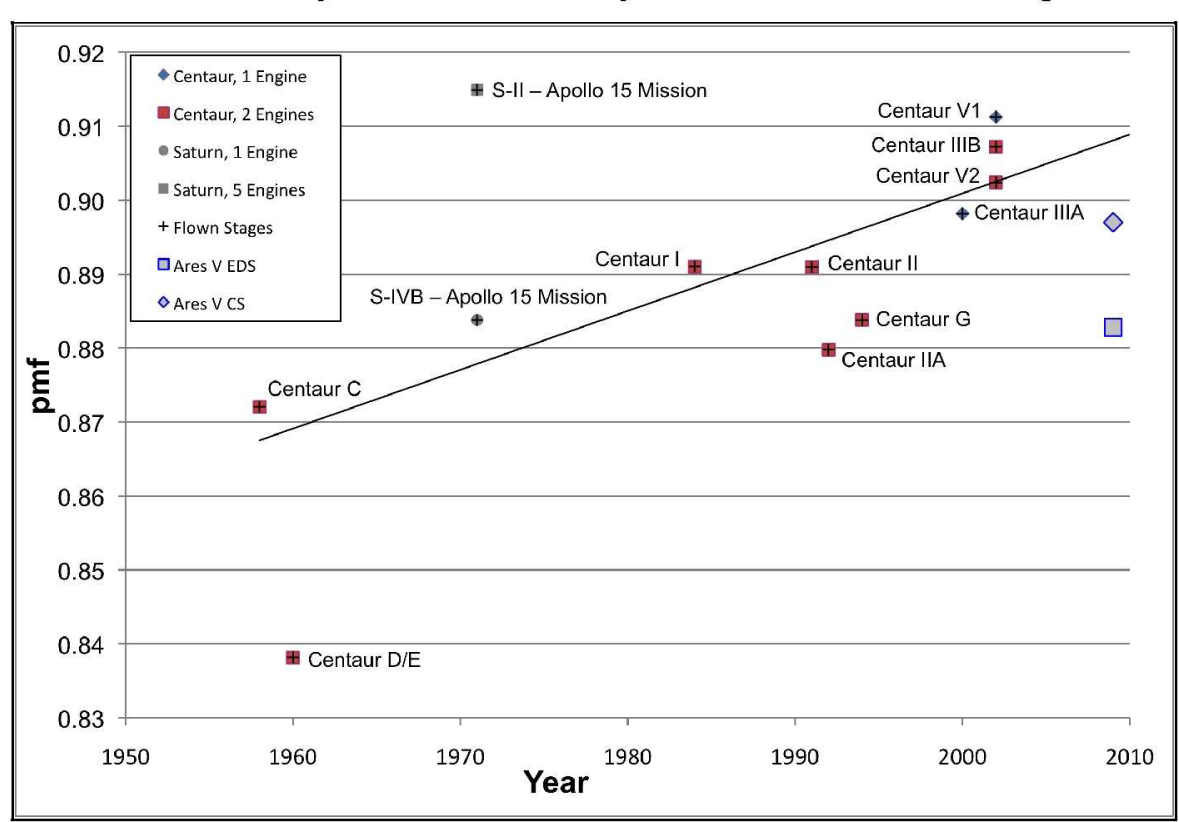

efficiency through advanced material development and improved designs) over time. Furthermore, Fig. 5 illustrates how far ahead of its time the Saturn S-II stage design was (depicted without the interstage included). Also, while the Ares V CS follows the trend reasonably well, the Ares V EDS is well below the trend line. This is indicative of the burdensome mission requirements of that stage.

Figure 5. Stage pmf trend. ${ }^{3-5}$

\section{Ares V Mission Requirements}

The EDS of the Ares $V$ launch vehicle has unique mission requirements that have a very large impact on its pmf and thus overall launch vehicle performance. This stage has approximately $558,000 \mathrm{lb}_{\mathrm{m}}$ of usable $\mathrm{LOX}^{\mathrm{L}} \mathrm{LH}_{2}$ propellant, and it expends about $60 \%$ of that propellant on Earth-to-orbit (ETO) ascent. However, it must remain in LEO for up to 4 days awaiting rendezvous with the CEV/Orion \& have the ability to restart its J-2X engine for the trans-lunar injection (TLI) maneuver. The 4 day loiter is a key driver for several subsystems on the EDS, including micrometeoroid/orbital debris (MMOD) protection, a loiter skirt for the placement of solar arrays or fuel cells, power distribution equipment, thermal radiators, cryogenic propellant boiloff while in orbit, and tank insulation to minimize the amount of cryogenic propellant boiloff.

In addition, the LCCR Ares V design allocates propellant for FPR and liquid residuals, and it includes a dry mass growth allowance based on percentages at a subsystem level. Overall, all of these design requirements effects a large impact on the EDS pmf. By removing these design requirements, the EDS pmf would increase from 0.883 to $\sim 0.923$, as seen in Table 3 . This would otherwise represent an approximately $35 \%$ increase in structural efficiency.

Table 3. Effect of mission requirements and design assumptions on pmf.

\begin{tabular}{|l|c|c|}
\hline \multicolumn{1}{|c|}{ LCCR 51.00.48 Vehicle } & Mass $\left(\mathbf{l b}_{\mathbf{m}}\right)$ & EDS pmf \\
\hline EDS Total GLOW & 632,000 & \\
\hline EDS Total Usable Propellant† & 558,000 & 0.883 \\
\hline \multicolumn{2}{|c|}{} & \\
\hline Design Parameters (cumulative) & 2,400 & 0.886 \\
\hline MMOD Shielding & 1,300 & 0.888 \\
\hline Loiter Skirt, Solar Arrays \& Power Distribution & 700 & 0.889 \\
\hline Propellant Boiloff (4 day loiter) & 800 & 0.890 \\
\hline Cryogenic Tank Insulation & 1,500 & 0.892 \\
\hline J-2X Engine Restart Propellant & 10,100 & 0.907 \\
\hline Flight Performance Reserve (FPR) & 4,700 & 0.914 \\
\hline Liquid Residuals & 5,800 & 0.923 \\
\hline Dry Mass Growth Allowance & & \\
\hline
\end{tabular}

† EDS total usable propellant is comprised of $330,000 \mathrm{lb}_{\mathrm{m}}$ of propellant used for ETO ascent and $228,000 \mathrm{lb}_{\mathrm{m}}$ used for the TLI maneuver. 


\section{Conclusion}

The calculation of the propellant mass fraction of a launch vehicle or the stage of a launch vehicle can vary slightly. If limited knowledge is available, very basic equations can be used to determine the approximate pmf. However, when more information is known or design assumptions require allocations of propellant for purposes other than propulsive maneuvers, those allocations should be discounted from the overall usable propellant mass. Furthermore, assumptions with regard to restarting engines, reserves, loiter skirts, power generation and distribution equipment, or dry mass growth can have a tremendous impact on the overall 'burnout' mass of the launch vehicle stage. It is imperative however, to include these design assumptions early in the design process to minimize performance risk later in the design cycle.

\section{References}

${ }^{1}$ D., Griffin, Michael. Space Vehicle Design. Washington, DC: AIAA, 1991. Print.

${ }^{2}$ Sutton, George, and Oscar Biblarz. Rocket Propulsion Elements. 7th ed. New York: John Wiley \& Sons, Inc., 2001. Print.

${ }^{3}$ Isakowitz, Steven J., Joshua B. Hopkins, and Joseph P. International Reference Guide to Space Launch Systems (General Publication). 4th ed. New York: AIAA, 2004. Print.

${ }^{4}$ Apollo 15 Flight Evaluation Report, NASA.

${ }^{5}$ NASA Ares Projects Office, Marshall Space Flight Center, AL. 


\section{Propellant Mass Fraction Calculation Methodology for Launch Vehicles and Application to Ares Vehicles}

\section{James B. Holt} NASA MSFC Advanced Concepts Office Timothy S. Monk NASA MSFC Ares Projects Office September $14^{\text {th }}, 2009$

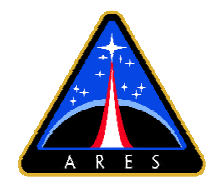




\section{Agenda}

Ares Family of Vehicles Overview

- Propellant Mass Fraction (pmf) Calculation Methods

- Fundamental pmf Calculation

- Usable Propellant Method

- Large Mass Exclusion Method

- Historical pmf Comparison

- Ares I Upper Stage (US) and Ares V Earth Departure Stage (EDS) comparison to flight-proven upper stages

- Ares V Core Stage (CS) comparison to flight-proven first stages

- pmf trend over time

- Ares V Mission Requirements

- Impact to pmf of various design parameters 


\section{Ares Vehicles Overview}
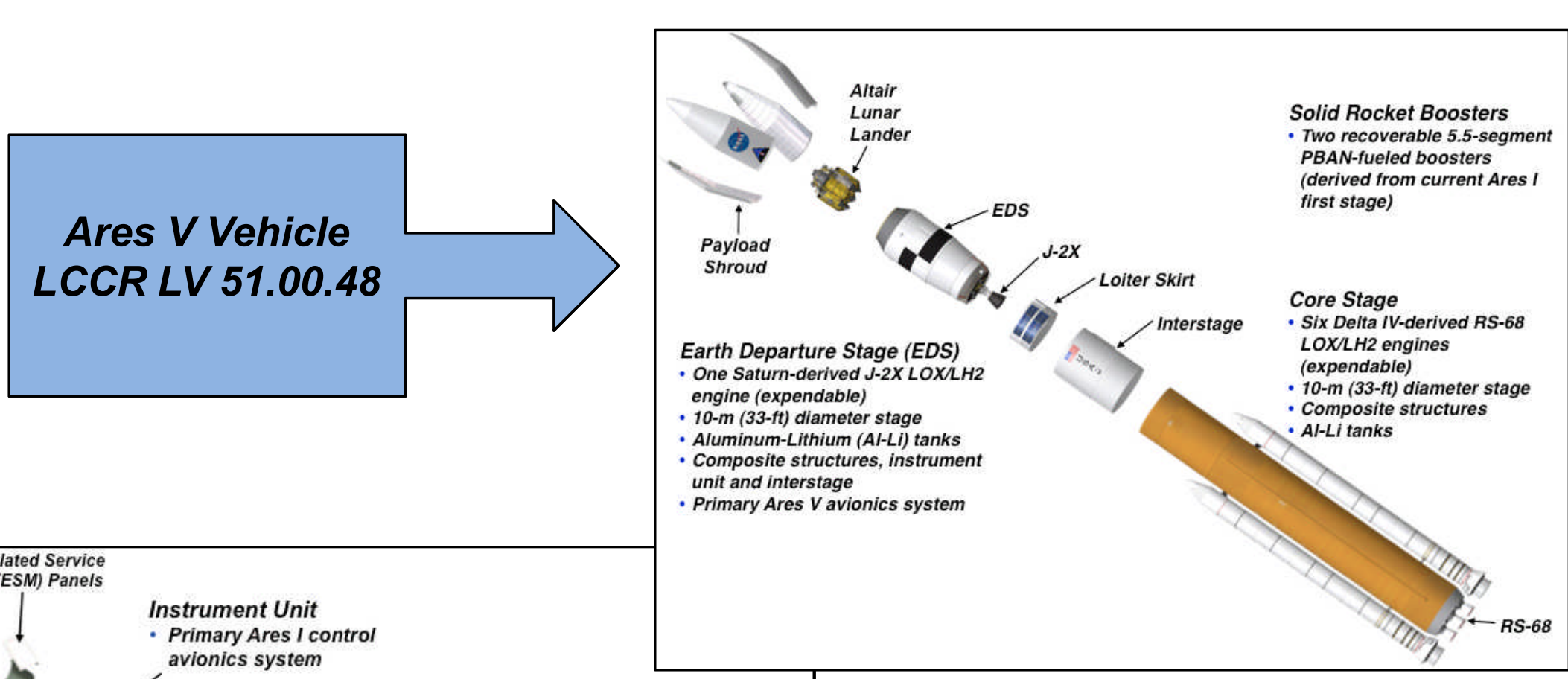

Upper Stage

- LOX/LH2 prop

- 5.5-m (18-ft) diameter

- Aluminum-Lithium (Al-Li) structures

Instrument Unit

- Primary Ares I control

Orion CEV Encapsulated Service

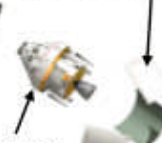

avionics system
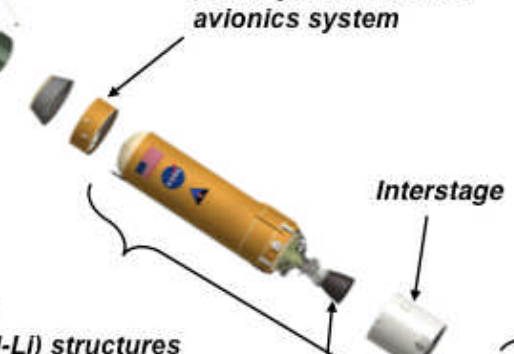

\section{First Stage}

- Derived from current

Shuttle RSRM/B

- Five segments/Polybutadiene

Acrylonitrile (PBAN) propellant - Recoverable

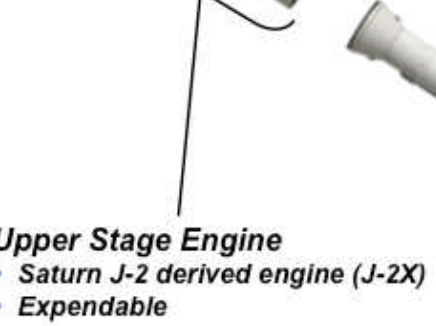

- Saturn $J-2$ derived engine $(J-2 X)$

- Expendable

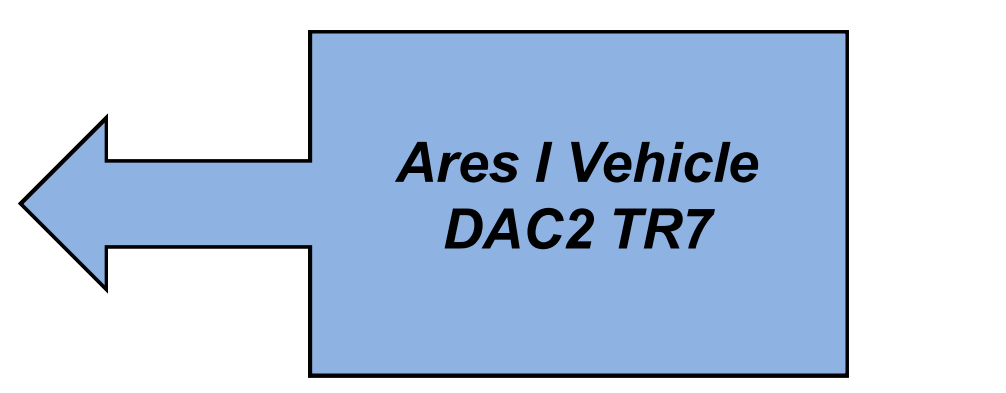




\section{Propellant Mass Fraction Calculation Methods}

- Fundamental Method:

- Uses only the total loaded propellant mass and dry mass of the stage

- Typically used when limited information is available

- For the LCCR 51.00.48 Ares V: 


\section{Propellant Mass Fraction Calculation Methods}

- Usable Propellant Method:

- Accounts for propellant allocated for several realistic conditions:

- Flight Performance Reserve (FPR)

- Fuel Bias

- Liquid Residuals

- Engine Restarts (if applicable)

- Purges/Bleeds (or venting)

- Boiloff of cryogenic propellants

- In-flight losses

- For the LCCR 51.00.48 Ares V: 


\section{Propellant Mass Fraction Calculation Methods}

- Large Mass Exclusion Method:

- Common when comparing stages that are similar with the exception of a single large mass discrepancy

- Engine Mass

- Interstage Mass

- Loiter Kits

- An example using the Saturn S-IVB \& Ares I US:

\begin{tabular}{|l|c|c|c|c|c|}
\hline & Usable Prop Mass $\left(\mathrm{Ib}_{\mathrm{m}}\right)$ & GLOM $\left(\mathrm{lb} \mathrm{m}_{\mathrm{m}}\right)$ & GLOM w/o Engine $\left(\mathrm{Ib}_{\mathrm{m}}\right)$ & $\mathrm{pmf}_{1}$ & pmf $_{2}$ \\
\hline Saturn S-IVB & $\sim 235,000$ & $\sim 266,000$ & $\sim 262,500$ & 0.884 & 0.895 \\
\hline Ares I US & $\sim 302,000$ & $\sim 344,000$ & $\sim 338,600$ & 0.878 & 0.892 \\
\hline
\end{tabular}

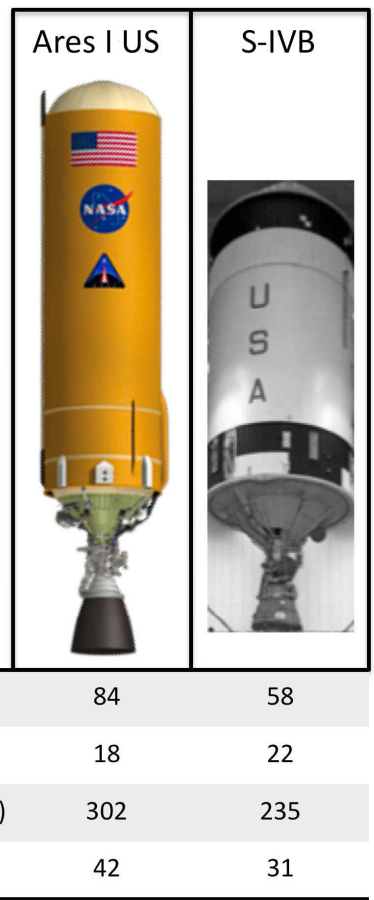




\section{Historical pmf Comparison - Upper Stages}

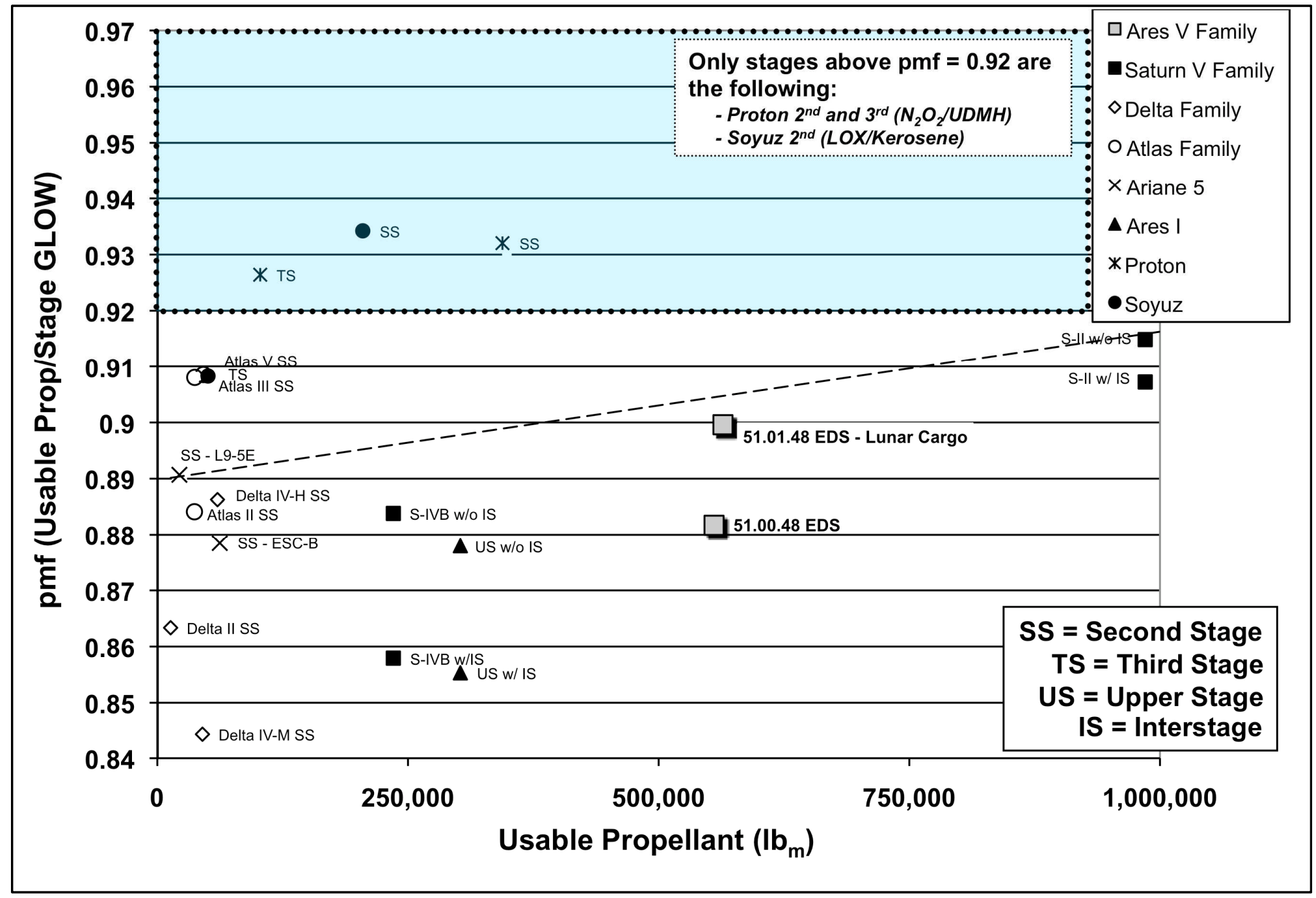

51.00.48 vs. 51.01.48 shows the impact of Ares V EDS loiter requirements on pmf 


\section{Historical pmf Comparison - First Stages}

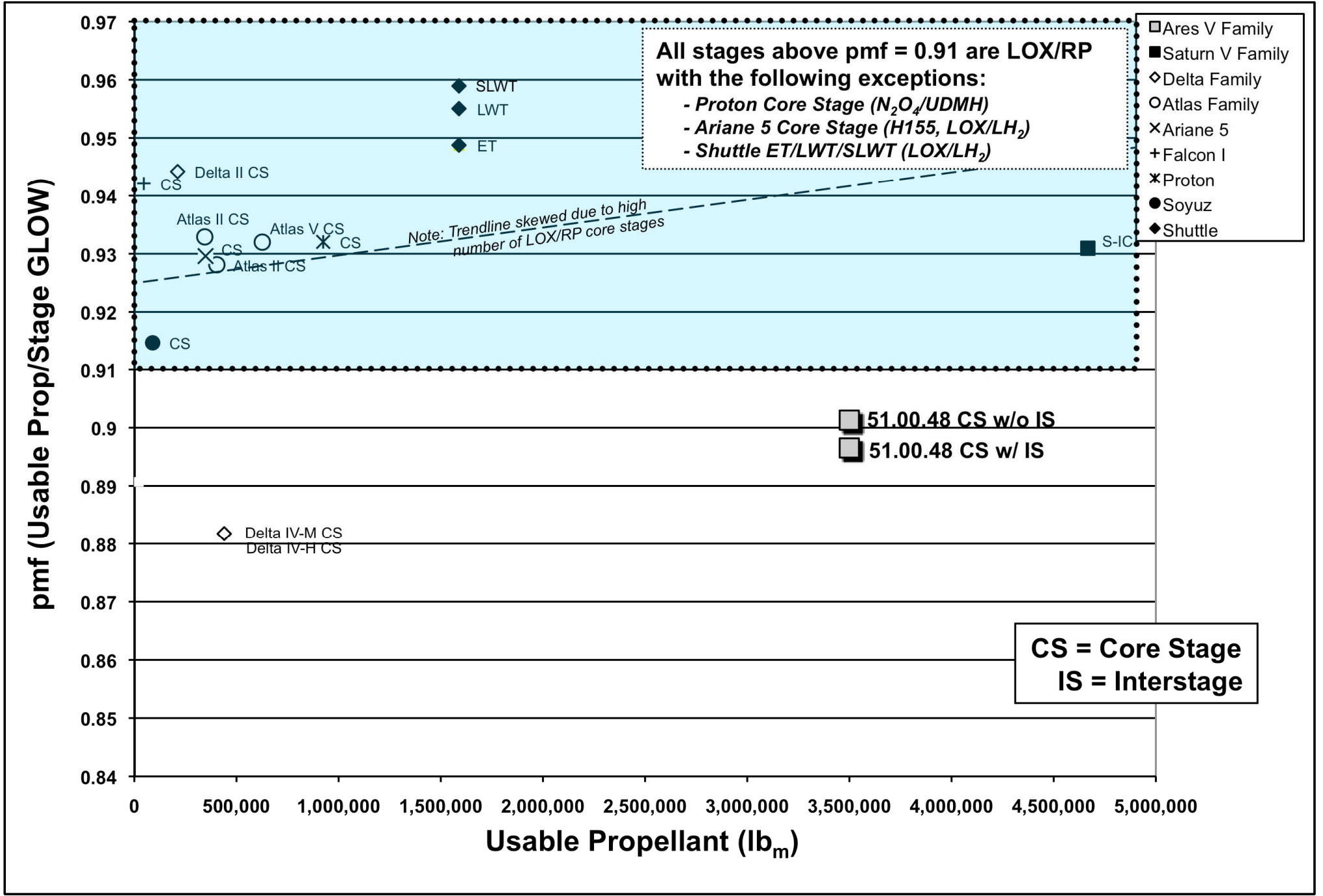




\section{pmf Trend}

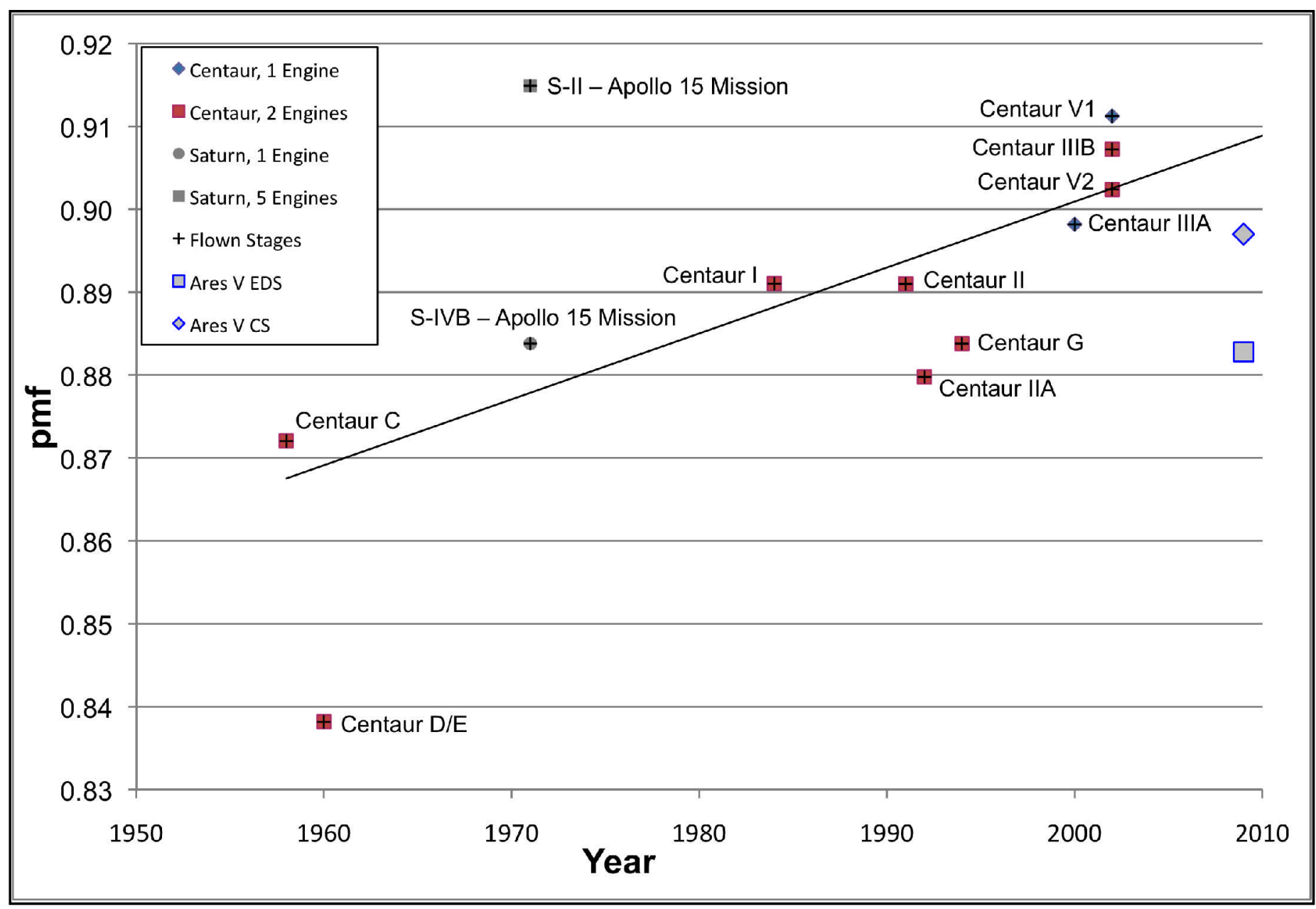

Ares V EDS appears to be overly conservative when compared to flown, domestic LOX/LH ${ }_{2}$ upper stages, but design requirements for that stage are very demanding 


\section{Ares V EDS Mission \& Design Requirements}

\begin{tabular}{|l|c|c|}
\hline \multicolumn{1}{|c|}{ LCCR 51.00.48 Vehicle } & Mass $\left.\mathbf{( I b}_{\mathbf{m}}\right)$ & EDS pmf \\
\hline EDS Total GLOW & 632,000 & \\
\hline EDS Total Usable Propellant & 558,000 & 0.883 \\
\hline \multicolumn{2}{|c|}{} & \\
\hline Design Parameters (cumulative) & & 0.886 \\
\hline MMOD Shielding & 2,400 & 0.888 \\
\hline Loiter Skirt, Solar Arrays \& Power Distribution & 1,300 & 0.889 \\
\hline Propellant Boiloff (4 day loiter) & 700 & 0.890 \\
\hline Cryogenic Tank Insulation & 800 & 0.892 \\
\hline J-2X Engine Restart Propellant & 1,500 & 0.907 \\
\hline Flight Performance Reserve (FPR) & 10,100 & 0.914 \\
\hline Liquid Residuals & 4,700 & 0.923 \\
\hline Dry Mass Growth Allowance & 5,800 & \\
\hline
\end{tabular}

- The 4 day loiter requirement is a major driver for the EDS design. This requirement has a large, negative impact on the stage pmf.

- In addition, design requirements for FPR, residuals, mass growth alllowance (MGA), etc. have a large, negative impact on stage pmf. 


\section{Conclusions}

- pmf calculation methods are very simple, but must be applied appropriately.

- pmf calculations can be used to provide a gauge of conservatism in a design, assess the mission/design requirements of a stage, or to compare candidate designs.

- Ares I US compares well historically to the S-IVB stage in terms of pmf.

- Ares V CS does not have a comparable stage for purposes of comparison, but it does compare reasonably well to the yearly trend of increases in pmf.

- Ares V EDS appears to have a low pmf (or overly conservative), but mission and design requirements must be considered. 